\title{
BMJ Open A quantitative analysis of the prevalence of clinical depression and anxiety in patients with prostate cancer undergoing active surveillance
}

\author{
Sam Watts, ${ }^{1}$ Geraldine Leydon, ${ }^{1}$ Caroline Eyles, ${ }^{1}$ Caroline M Moore, ${ }^{2}$ \\ Alison Richardson, ${ }^{3}$ Brian Birch, ${ }^{4}$ Philip Prescott, ${ }^{5}$ Catrin Powell, ${ }^{6}$ George Lewith ${ }^{1}$
}

To cite: Watts S, Leydon $\mathrm{G}$, Eyles $\mathrm{C}$, et al. A quantitative analysis of the prevalence of clinical depression and anxiety in patients with prostate cancer undergoing active surveillance. BMJ Open 2015;5:e006674

doi:10.1136/bmjopen-2014006674

- Prepublication history for this paper is available online. To view these files please visit the journal online (http://dx.doi.org/10.1136/ bmjopen-2014-006674).

Received 23 September 2014 Revised 13 March 2015 Accepted 18 March 2015

CrossMark

For numbered affiliations see end of article.

Correspondence to

Sam Watts;

sw1u09@soton.ac.uk

\section{ABSTRACT}

Objective: To quantitatively determine the prevalence of anxiety and depression in men on active surveillance (AS).

Design: Cross-sectional questionnaire survey.

Setting: Secondary care prostate cancer (PCa) clinics across South, Central and Western England.

Participants: 313 men from a total sample of 426 with a histological diagnosis of PCa currently managed with AS were identified from seven UK urology departments. The mean age of respondents was 70 (51-86) years with the majority $(76 \%)$ being married or in civil partnerships. $94 \%$ of responders were of white British ethnicity.

Primary outcome measures: The prevalence of clinically meaningful depression and anxiety as assessed by the Hospital Anxiety and Depression Scale (HADS; score $\geq 8 / 21$ ).

Secondary outcome measures: Patient demographic data (age, employment, relationship, ethnic and educational status). Each demographic variable was cross-tabulated against patients identified as depressed or anxious to allow for the identification of variables that were significantly associated with depression and anxiety. In order to determine predictors for depression and anxiety among the demographic variables, logistic regression analyses were conducted, with $p<0.05$ considered as indicating statistical significance.

Results: The prevalence of clinical anxiety and depression as determined via the HADS (HADS $\geq 8$ ) was $23 \%(n=73)$ and $12.5 \%(n=39)$, respectively. Published data from men in the general population of similar age has shown prevalence rates of $8 \%$ and $6 \%$, respectively, indicating a twofold increase in depression and a threefold increase in anxiety among AS patients. Our findings also suggest that AS patients experience substantially greater levels of anxiety than patients with $\mathrm{PCa}$ treated radically. The only demographic predictor for anxiety or depression was divorce.

Conclusions: Patients with PCa managed with AS experienced substantially higher rates of anxiety and depression than that expected in the general population. Strategies to address this are needed to improve the management of this population and their quality of life.

\section{Strengths and limitations of this study}

- This is the largest multicentre assessment of depression and anxiety prevalence in active surveillance patients yet to be undertaken.

- Our cross-sectional methodology makes it difficult to draw definitive conclusions about the causality of anxiety and depression in this population, and how these conditions vary over time.

- Our anonymous recruitment policy means that we are unable to account for nor determine differences between responders and nonresponders.

\section{INTRODUCTION}

Prostate cancer $(\mathrm{PCa})$ represents the most common form of non-cutaneous malignancy diagnosed in British men. ${ }^{1}$ The number of men being diagnosed with PCa continues to rise annually as a result of increased PSA testing and an ageing population. ${ }^{2}$ Men with a diagnosis of low-risk PCa face a decision between radical treatments such as surgery and brachytherapy, and active surveillance (AS).

AS aims to personalise the management of PCa by ensuring that only those men with clinically relevant disease are selected for curative treatment. Analysis of several large case series suggests that around four in five men will remain untreated at 2 years, with a recent UK series reporting adverse histology of $22 \%$ at a median of 5.7 years, with treatment-free probability of $70 \%$ at that time point. ${ }^{3}$

There is doubt about the utility of radical surgery for men with low-risk disease as it has been shown to have no significant benefit on 12-year survival. ${ }^{4}$ Radical surgery is also associated with significantly greater risk of longterm incontinence $(17.1 \%)$ and impotence $(81.1 \%)$ than $\mathrm{AS}^{4}$ The recently updated (2014) UK National Institute for Health and 
Care Excellence (NICE) guidelines currently recommend AS as the preferred management option for men with low-risk localised PCa. ${ }^{5}$

While the maintenance of physical and sexual function is a major advantage of AS, little is currently known about the psychological consequences of this strategy. Much research has highlighted the psychological benefits of early active treatment initiation among patients with cancer. ${ }^{6}{ }^{7}$ Benefits include feelings of empowerment, hope and reassurance. ${ }^{6-8}$ It has been proposed that men who choose AS may be at greater risk of psychological distress because of the passive nature of the strategy. ${ }^{9}$

Burnet $e a^{10}$ assessed psychological distress in 100 men on AS in a single-centre UK study. They reported a high prevalence of anxiety and a low prevalence of depression (21\% and $4 \%$, respectively). Van den Bergh et $a l^{11}{ }^{12}$ assessed depression and anxiety in 129 men on AS from the Prostate Cancer Research International (PRIAS) study, and also reported a low prevalence of depression $(8 \%)$ and a high prevalence of anxiety $(17 \%)$.

These findings suggest that the prevalence of anxiety in this patient population may be two to three times greater than that found in men of similar age in the general population. ${ }^{13}$ Crucially, anxiety is known to be a significant predictor in identifying which men on AS will transfer to radical treatment in the absence of a change in histology or PSA. ${ }^{14} 15$ The assessment and management of anxiety in this patient population should, therefore, be viewed as an area of key clinical importance to help prevent this transference and the debilitating physical side effects that accompany it.

The primary aim of the current investigation was to address this issue by undertaking a large cross-sectional assessment of the prevalence of clinical anxiety and depression in a large cohort of AS patients from multiple centres across the UK. It was hoped that by doing so we would provide a geographically diverse, ethnically varied, clinically relevant and more definitive estimate of the psychological health of this patient group. A secondary aim was to determine whether any demographic variables were able to predict which AS patients were most likely to experience depression and anxiety.

\section{METHODS}

The coordinating site for the running of this study was Southampton University Hospitals Trust. Six additional National Health Service (NHS) Trust sites (Great Western, Mid-Essex, Coventry and Warwickshire, Weston Area Foundation, University College London Hospitals, and Surrey and Sussex NHS Trust Foundations) were recruited as secondary sites. Sponsorship was obtained by Southampton University Hospitals Trust.

Participants were recruited consecutively over a 7-month period from May 2012 to December 2012. To be eligible for entry into the study, each participant had to meet all of the following criteria which were standardised across all seven recruitment centres:
- A biopsy-confirmed diagnosis of PCa.

- Diagnosis received at least 2 months prior to entry into the study to minimise acute, postdiagnosis mood disturbances.

- Currently being managed with AS.

- Be fluent in the English language (questionnaires were written and validated in English).

- Have no additional cancer diagnoses.

- Have no other serious or life-threatening comorbidity that could significantly impact on mood such as late-stage cardiovascular disease, kidney failure or stroke.

To help maximise recruitment, all patients meeting the inclusion criteria were eligible for entry into the study. No restrictions or parameters were put in place in terms of patient age or time since diagnosis. Records at each of the seven centres were screened to identify patients who met the above inclusion criteria. All eligible patients were then sent by post a Patient Information Pack (PIP). The PIP included a Patient Invitation Letter, a Hospital Anxiety and Depression Scale $\left(\operatorname{HADS}^{16}\right)$, a Patient Demographic Questionnaire (PDQ) and a freepost return envelope. Participants wishing to take part in the investigation were asked to anonymously complete and return both of the enclosed questionnaires. An anonymous recruitment policy was adopted based on the advice of Berkshire Research Ethics Committee. While doing so safeguarded patient identity and security, it did mean that we were unable to identify responders and non-responders, nor screen their records to collect relevant clinical data on issues such as date of diagnosis and a history of psychological morbidity.

The HADS is a well-validated and reliable questionnaire that has been used extensively within the field of oncology to assess depression and anxiety. ${ }^{17-20}$ Seven questions relate to symptoms of depression and seven to anxiety. The maximum score is 21 for both the anxiety and depression subscales. In the current investigation, depression and anxiety were deemed to exist in patients with a HADS score of $\geq 8$, based on the recommendations of Zigmond and Snaith. ${ }^{16}$ The PDQ was developed by the research team at the University of Southampton to measure the key demographic information required for this study (age, ethnic, employment, relationship and educational status).

Data from the returned questionnaires were transferred to SPSS V.19.0 for analysis. Descriptive statistics were computed to estimate the percentage prevalence of depression and anxiety (\% of participants scoring $\geq 8$ on each of the HADS subscales). Each of the collected demographic variables were cross-tabulated against patients identified as depressed or anxious to allow for the identification of any variables that were significantly associated with depression and anxiety. Finally, in order to determine predictors for depression and anxiety among the demographic variables, logistic regression analyses were conducted, with $\mathrm{p}<0.05$ considered as indicating statistical significance. 


\section{RESULTS}

Three hundred and thirteen participants out of an approached sample of $426(73.47 \%)$ were recruited from seven NHS Trust sites into the current investigation (table 1). A sample of this size provided sufficient precision to estimate the percentage of men who were depressed and anxious using a $95 \%$ CI within $4 \%$ of the true value. The mean age of the patients was 70.49 years (range 51-86). Most participants were retired (74.44\%) and married/in civil partnership (76.99\%); 36.6\% had completed their education to secondary school level, while $32.3 \%$ completed college/specialised training. The majority of participants $(93.8 \%)$ were of white British ethnicity. Eight men did not disclose their age, and five did not complete the remainder of the demographic questions.

Across the 313 participants, the mean depression score was $3.269(\mathrm{SD}=3.569)$, with scores ranging from 0 to 18 . Thirty-nine participants $(12.5 \%)$ obtained a score of $\geq 8$ on the HADS-D, indicating the existence of clinical depression. The mean anxiety score across the 313 participants was $4.84(\mathrm{SD}=3.791)$, with scores ranging from 0 to 20. Seventy-three participants (23.3\%) recorded a score of $\geq 8$ on the HADS-A, indicating clinical anxiety (table 2 ).

Only one cross-tabulation of demographic variables against depressed or anxious cases showed a significant variation in the prevalence of either condition around the overall estimates (relationship status vs anxiety). Although very few divorced men were included in the study, this finding suggests that divorced patients have a higher prevalence of anxiety $(56 \%)$ compared with the overall rate of $23.1 \%$ (table 3 ).

\section{PREDICTORS OF DEPRESSION AND ANXIETY Depression}

Each demographic variable was used as a possible predictor to determine whether it could be included in the logistic regression model to improve its predictive value. A stepwise analysis was utilised for this purpose where the $\mathrm{p}$ value for entry was taken by default as 0.05 . The results indicated that 'Southampton' was the best single predictor with a $\mathrm{p}$ value $<0.0005$. None of the other

\begin{tabular}{|c|c|c|c|c|c|c|c|c|}
\hline Site & S'ton & Swindon & Essex & Coventry & $\begin{array}{l}\text { Weston- } \\
\text { S-Mare }\end{array}$ & UCLH & $\begin{array}{l}\text { Sussex and } \\
\text { Surrey }\end{array}$ & All \\
\hline Number of responders & 28 & 89 & 14 & 92 & 15 & 23 & 52 & 313 \\
\hline \multicolumn{9}{|l|}{ Age (years) } \\
\hline Mean (SD) & $\begin{array}{l}73.0 \\
(8.598)\end{array}$ & $\begin{array}{l}71.9 \\
(7.276)\end{array}$ & $\begin{array}{l}66.5 \\
(2.953)\end{array}$ & $\begin{array}{l}67.93 \\
(5.441)\end{array}$ & $\begin{array}{l}77.93 \\
(5.849)\end{array}$ & $\begin{array}{l}68.13 \\
(5.684)\end{array}$ & $\begin{array}{l}68.08 \\
(5.577)\end{array}$ & $\begin{array}{l}70.49 \\
(7.335)\end{array}$ \\
\hline Range & $55-86$ & $53-72$ & $62-72$ & 50-79 & $71-81$ & $51-77$ & $50-81$ & $51-86$ \\
\hline \multicolumn{9}{|l|}{ Employment status } \\
\hline Full-time employment & 4 & 10 & 0 & 16 & 0 & 7 & 4 & 41 \\
\hline Part-time employment & 4 & 5 & 4 & 3 & 0 & 6 & 6 & 28 \\
\hline Retired & 19 & 72 & 7 & 69 & 15 & 10 & 42 & 234 \\
\hline Unemployed & 1 & 1 & 0 & 3 & 0 & 0 & 0 & 5 \\
\hline Unknown & 0 & 1 & 3 & 1 & 0 & 0 & 0 & 5 \\
\hline \multicolumn{9}{|l|}{ Relationship status } \\
\hline Co-habiting & 2 & 5 & 0 & 1 & 0 & 2 & 4 & 14 \\
\hline Divorced & 4 & 0 & 1 & 5 & 2 & 2 & 2 & 16 \\
\hline Married/civil partnership & 18 & 70 & 10 & 75 & 9 & 17 & 40 & 239 \\
\hline Single & 0 & 2 & 0 & 7 & 0 & 2 & 5 & 16 \\
\hline Widowed & 4 & 11 & 0 & 3 & 4 & 0 & 1 & 23 \\
\hline Unknown & 0 & 1 & 3 & 1 & 0 & 0 & 0 & 5 \\
\hline \multicolumn{9}{|l|}{ Education status } \\
\hline Left school before age 15 & 3 & 23 & 0 & 18 & 4 & 0 & 8 & 56 \\
\hline Completed secondary education & 18 & 27 & 6 & 27 & 6 & 5 & 17 & 106 \\
\hline College/specialised training & 6 & 31 & 3 & 34 & 4 & 5 & 16 & 99 \\
\hline University & 2 & 7 & 2 & 12 & 1 & 13 & 10 & 47 \\
\hline Unknown & 0 & 1 & 3 & 1 & 0 & 0 & 0 & 5 \\
\hline \multicolumn{9}{|l|}{ Ethnicity } \\
\hline White British & 28 & 85 & 11 & 87 & 15 & 19 & 51 & 296 \\
\hline White Other & 0 & 1 & 0 & 3 & 0 & 2 & 0 & 6 \\
\hline Afro-Caribbean & 0 & 2 & 0 & 0 & 0 & 2 & 0 & 4 \\
\hline Asian & 0 & 0 & 0 & 2 & 0 & 0 & 1 & 3 \\
\hline Other & 0 & 1 & 0 & 0 & 0 & 0 & 0 & 1 \\
\hline Unknown & 0 & 0 & 3 & 0 & 0 & 0 & 0 & 3 \\
\hline
\end{tabular}


Table 2 Mean Hospital Anxiety and Depression Scale (HADS) scores and prevalence data

\begin{tabular}{llllllll}
\hline & Mean (with 95\% Cls) & SE & Median & SD & Minimum & Maximum & Caseness (HADS $\geq 8$ ) \\
\hline HADS-D (depression) & $3.269(2.9$ to 4.3$)$ & 0.281 & 2 & 3.569 & 0 & 18 & $\mathrm{~N}=39(12.46 \%)$ \\
HADS-A (anxiety) & $4.84(4.4$ to 5.3$)$ & 0.299 & 4 & 3.791 & 0 & 20 & $\mathrm{~N}=73(23.32)$ \\
\hline
\end{tabular}

variables were significant with the next lowest $p$ value being 0.119 for 'Single' (table 4).

Table 5 shows the estimated model parameters at step 1 of the logistic regression with only Southampton included, and also those for the logistic regression with Southampton already in the model and the variable Single entered as the next most significant predictor, with a $p$ value of 0.055 .

The OR for Southampton was 4.904 and 5.422 for both steps 1 and 2, indicating that there is a higher chance of being depressed among the men from Southampton. When these variables were included in the logistic regression model in turn and the probability of being a depressed man was evaluated for each patient (table 6) none of the probabilities were increased sufficiently to change the proportion of correctly classified men. The cut-off value for the estimated probability of being depressed was taken to be 0.5 .

\section{Anxiety}

The logistic regression analysis performed for depression was repeated to investigate predictions of anxiety using the demographic variables. Each variable used as a possible predictor was assessed to see whether it could be included in the model to improve its predictive value (table 7 ).

Divorce was the only significant variable and was added into the model. The logistic regression parameter estimates for predicting anxiety using divorced were shown to be insignificant (table 8).

\section{DISCUSSION}

We observed a high prevalence of depression and anxiety in men with PCa being managed with AS, but were unable to identify any clear demographic predictors for these conditions other than being divorced. Based on the most recent UK statistics, the prevalence of depression and anxiety in British men aged over 65 is $6 \%$ and $8 \%$, respectively. ${ }^{13}$ Our results from the HADS suggest that AS patients may be more than twice as likely to be depressed and three times as likely to be anxious, compared with men of similar age in the community. Across the 313 recruited AS patients, the mean scores of depression and anxiety were low at 3.27 (SD 3.33) and 4.84 (SD 3.85), respectively. However, using the standardised threshold of $\geq 8$ on the HADS to define caseness, $12.5 \% \quad(n=39)$ of participants met the criteria for clinically meaningful depression and $23.3 \%(n=73)$ for anxiety.

AS patients who were divorced had a significantly higher prevalence of anxiety. None of the additional demographic variables had any significant power to predict which men were most likely to be experiencing depression or anxiety. Such findings suggest that the men defined as depressed and anxious in this study were a demographically heterogeneous group. Other smaller studies have identified that variables such as impaired mental health and the patient's perceived importance of the physician in the treatment decisionmaking process appear to be associated with poorer quality of life among AS patients. ${ }^{11} 21$ This would suggest that variables other than the simple data set collected in our current study may be able to predict which AS patients are most vulnerable to psychological distress. Further research is needed to help clarify this situation.

Several important limitations exist within the current study. We did not recruit a cohort of patients with PCa who were receiving radical treatment. We are, therefore, unable to determine how the prevalence rates of HADS-based depression and anxiety observed in the AS patients compare to those seen in patients receiving radical treatment. The only previous study that has attempted to answer this question reported that AS patients experienced over twice the prevalence of anxiety and an equal prevalence of depression as radically treated patients. ${ }^{10}$

Likewise, while our findings suggest that AS patients experience a much greater prevalence of anxiety and depression when compared with men of a similar age without $\mathrm{PCa},{ }^{13}$ our conclusions are drawn from indirect comparisons with previously conducted research rather

Table 3 Anxious versus relationship cross-tabulation

\begin{tabular}{lllllcc}
\hline & Co-habiting & Divorced & Married & Single & Widowed & Total \\
\hline Non-anxious $(\mathrm{n})$ & 10 & 7 & 187 & 12 & 21 & 237 \\
Within relationship (\%) & 71.4 & 43.8 & 78.2 & 75 & 91.3 & 76.9 \\
Anxious (n) & 4 & 9 & 52 & 4 & 2 & 71 \\
Within relationship (\%) & 28.6 & 56.3 & 21.8 & 25 & 23.7 & 23 \\
Total & 14 & 16 & 239 & 16 & 308 \\
\hline$\chi^{2}=13.114, \mathrm{p}=0.011$. & & & & &
\end{tabular}


Table 4 Significant predictors of depression

\begin{tabular}{lcll}
\hline Included levels & Score & df & Significance \\
\hline Full time & 0.003 & 1 & 0.956 \\
Part time & 0.821 & 1 & 0.365 \\
Retired & 1.547 & 1 & 0.214 \\
Semiretired & 0.143 & 1 & 0.706 \\
Unemployed & 0.584 & 1 & 0.445 \\
Co-habiting & 0.045 & 1 & 0.832 \\
Divorced & 0 & 1 & 0.996 \\
Married & 0.514 & 1 & 0.473 \\
Single & 2.431 & 1 & 0.119 \\
Widowed & 1.498 & 1 & 0.221 \\
Asian & 0.287 & 1 & 0.594 \\
BA & 0.143 & 1 & 0.706 \\
BC & 0.431 & 1 & 0.511 \\
Chinese & 0.143 & 1 & 0.706 \\
WB & 0.443 & 1 & 0.505 \\
WO & 0.099 & 1 & 0.753 \\
College & 0.265 & 1 & 0.607 \\
Specialist college & 0.596 & 1 & 0.44 \\
Left school <15 & 0.129 & 1 & 0.719 \\
Secondary & 0.215 & 1 & 0.643 \\
University & 0.701 & 1 & 0.403 \\
Coventry & 1.693 & 1 & 0.193 \\
Essex & 1.081 & 1 & 0.299 \\
London & 0.323 & 1 & 0.57 \\
SASH & 1.3 & 1 & 0.254 \\
Southampton & 15.245 & 1 & 0 \\
Swindon & 0.119 & 1 & 0.73 \\
Western-S-M & 2.243 & 1 & 0.134 \\
\hline df, degrees of freedom. & & & \\
& & &
\end{tabular}

than from direct comparisons with an age-matched control group. Furthermore, directly comparing clinical populations with population-based controls is not always a legitimate form of comparison for a variety of reasons, including biases such as Berkson's bias. To address this is would be helpful for future research to assess how the prevalence of depression and anxiety among AS patients varies in comparison to populations of patients with $\mathrm{PCa}$ treated radically.

Similarly, the depression and anxiety prevalence data generated in this study were collected by the HADS. However, as the HADS excludes any somatic symptoms of depression and anxiety, our definition of these conditions are not consistent with the common clinical definitions of generalised anxiety disorder and major depressive disorders, which all include somatic symptoms. There, therefore, exists an important discrepancy between our definition of depression and anxiety in this patient cohort, and that based on a clinical diagnosis that utilises somatic symptomology.

As a cross-sectional survey, we were also unable to assess how the prevalence of depression and anxiety may have altered over time. Anxiety tends to peak among AS patients 3-4 weeks prior to PSA testing and subsides substantially, if the subsequent clinical outcome indicates a lack of disease progression. ${ }^{22}$ The cross-sectional nature of the current investigation, coupled with the required anonymised recruitment process, made it impossible to determine where each patient was with regard to both their PSA monitoring schedule and how long ago were they diagnosed. Both of these issues may possibly have had some impact on the survey outcome, particularly as time from diagnosis has been shown to be a significant predictor of maladaptive adjustment to $\mathrm{PCa}^{21}$ However, as a cross-sectional survey, this investigation was not designed to determine causality or longitudinal changes in psychological morbidity, but did additional longitudinal investigations that assess depression and anxiety at the time of diagnosis up to the selection of AS, and therefore, the subsequent long-term follow-up are vital to help us address this issue.

The anonymous recruitment process that we adopted at the request of our local Research Ethics Committee meant that we were unable to account for a history of depression and anxiety. Depressed and anxious patients with cancer are less likely to complete and return questionnaires than their non-depressed and non-anxious counterparts. ${ }^{23}$ It is, therefore, possible that the prevalence data generated represent an underestimate of distress. This limits the generalisabilty of our findings.

Similarly, the anonymous recruitment process meant that we were unable to compare how responders differed from non-responders both clinically and demographically. It also meant that we were unable to initiate a second round of recruitment in a bid to recruit a subgroup of responders from the overall pool of nonresponders. While this limits the generalisability of our findings, it was a decision driven by the requirements of our governing Research Ethics Committee.

Lastly, our finding that divorced patients experienced a higher prevalence of anxiety was interesting. However,

Table 5 Logisitic regression for depression with 95\% Cls

\begin{tabular}{lrlrllll}
\hline & B & SE & Wald & df & Significance & OR & 95\% Cls \\
\hline Step 1 & & & & & & & \\
$\quad$ Southampton & 1.590 & 0.440 & 13.037 & 1 & 0.000 & 4.904 & 2.07 to 11.62 \\
$\quad$ Constant & -2.178 & 0.196 & 123.555 & 1 & 0.000 & 0.113 & \\
Step 2 & & & & & & & \\
$\quad$ Single & 1.180 & 0.614 & 3.687 & 1 & 0.055 & 3.253 & 0.97 to 10.84 \\
$\quad$ Southampton & 1.691 & 0.447 & 14.314 & 1 & 0.000 & 5.422 & 2.26 to 13.02 \\
$\quad$ Constant & -2.278 & 0.210 & 117.705 & 1 & 0.000 & 0.102 & \\
dff, degrees of freedom. & & & & & & &
\end{tabular}


Table 6 Classification of depressed men with the inclusion of Southampton and single

\begin{tabular}{lll}
\hline Level & Observed & $\begin{array}{l}\text { Percentage } \\
\text { correct }\end{array}$ \\
\hline Soton & Non-depressed $(n=274)$ & 100 \\
& Depressed $(n=39)$ & 0 \\
& Overall per cent & 87.50 \\
Sotassification & \\
single & Non-depressed $(n=274)$ & 100 \\
& Depressed $(n=39)$ & 0 \\
& Overall per cent & 87.50 \\
& classification & \\
\hline
\end{tabular}

it is important to note that the observed association between anxiety and divorce may more accurately reflect association with the incidence of PCa.

Important strengths of the current study include its multicentre recruitment, large sample size and high response rate which are very likely to have minimised the effect of any of the above potential confounders. Previous investigations into the prevalence of psychological distress in AS patients have involved small patient samples ${ }^{10-12}$ which substantially limits the representativeness and generalisability of their findings. Our results, therefore, provide a more robust and confident assessment of psychological distress. Furthermore, previous investigations of AS patients have typically recruited

\begin{tabular}{lccl} 
Table 7 & Significant predictors of anxiety & \\
\hline Full time & 0.993 & 1 & 0.334 \\
Part time & 0.048 & 1 & 0.826 \\
Retired & 2.943 & 1 & 0.086 \\
Semiretired & 3.298 & 1 & 0.069 \\
Unemployed & 1.612 & 1 & 0.204 \\
Co-habiting & 0.226 & 1 & 0.635 \\
Divorced & 10.223 & 1 & 0.001 \\
Married & 1.385 & 1 & 0.239 \\
Single & 0.027 & 1 & 0.871 \\
Widowed & 2.97 & 1 & 0.085 \\
Asian & 0.612 & 1 & 0.434 \\
BA & 3.298 & 1 & 0.069 \\
BC & 0.921 & 1 & 0.337 \\
Chinese & 0.305 & 1 & 0.581 \\
WB & 0.373 & 1 & 0.542 \\
WO & 0.343 & 1 & 0.558 \\
College & 0.031 & 1 & 0.859 \\
Specialist college & 0.158 & 1 & 0.691 \\
Left school <15 & 0.058 & 1 & 0.809 \\
Secondary & 0.009 & 1 & 0.923 \\
University & 0.426 & 1 & 0.514 \\
Coventry & 0.52 & 1 & 0.471 \\
Essex & 3.127 & 1 & 0.077 \\
London & 0.702 & 1 & 0.402 \\
SASH & 1.262 & 1 & 0.261 \\
Southampton & 1.338 & 1 & 0.247 \\
Swindon & 0.05 & 1 & 0.822 \\
Western-S-M & 0.097 & 1 & 0.755 \\
\hline
\end{tabular}

patients from single sites which further limits their generalisabilty. In the current investigation, men were recruited from seven independent NHS Trust locations across South, Western and Central England and thus, increased both patient diversity and generalisabilty as well as allowing us to investigate some demographic predictors.

Nevertheless, over $95 \%$ of participants were of white British origin representing an extremely homogeneous ethnic sample. This means we have very little understanding of how men from other ethnic communities may respond and cope with a PCa diagnosis and subsequent management with AS.

The work by Burnet et $a l^{10}$ compared men who were either currently receiving radical treatment with radiotherapy+neoadjuvant hormone therapy, or who had previously received radical radiotherapy with those on AS. Burnet $e t a l^{10}$ suggest a lack of significant difference in psychological morbidity between patients with PCa treated radically and those being managed with AS. However, those being managed with AS had over twice the prevalence of anxiety as those who were currently receiving treatment (21\% vs $10 \%)$, with $15 \%$ of men who had completed treatment exceeding the threshold for clinically relevant anxiety. The patient numbers involved meant this factor did not emerge as a statistically significant observation.

Our findings appear to be consistent with those generated from smaller investigations into psychological distress among AS patients. ${ }^{10-12}$ These investigations have all shown a lower prevalence of depression $(<10 \%)$ and a higher prevalence of anxiety $(17-21 \%)$. This was a pattern very clearly mirrored in the current study. In light of our large multicentre sample size, our findings would appear to support the initial conclusions from these previous, smaller investigations.

Similarly, our findings provide a unique insight into how the prevalence of psychological distress in AS patients compares to that observed among patients with PCa treated radically. Recently published meta-analysis data collected from a pooled sample of over 4000 patients with PCa have revealed a depression and anxiety prevalence of $14.70 \%$ (95\% CI $11.92 \%$ to $17.99 \%$ ) and $15.09 \%$ (95\% CI $12.15 \%$ to $18.60 \%$ ), respectively, among patients with PCa undergoing radical treatment. ${ }^{24}$ The findings of the current study, therefore, suggest that men managed with AS experience a slightly lower prevalence of depression and a substantially higher prevalence of anxiety than men treated radically. This is an important finding as it is the first large multicentre study to highlight the potential anxiety inducing nature of AS when used as a management approach for localised PCa.

In conclusion, we have identified the presence of considerable distress in this population, and the consequent need for the development and implementation of frameworks that will allow for the better management of anxiety in men receiving AS. To be effective, it is likely that such interventions will need to target and address AS patient's health literacy to allow them to better 
Table 8 Logistic regression analysis for anxiety with $95 \% \mathrm{Cls}$

\begin{tabular}{lllllllll}
\hline & B & SE & Wald & df & Significance & OR & 95\% Cls \\
\hline Step 1 & Divorced & 1.543 & 0.523 & 8.698 & 1 & 0.303 & 4.681 & 1.681 to 13.055 \\
& Constant & -1.292 & 0.141 & 83.832 & 1 & 0.000 & 0.275 & \\
\hline df, degrees of freedom. & & & & & & &
\end{tabular}

understand, process, rationalise AS and thus, minimise their anxiety.

Self-care interventions that equip patients with PCa with psychological, educational and informational support, either remotely via the internet or in groupbased environments, have shown some initial success in lowering psychological distress and improving healthrelated quality of life. ${ }^{25}$ To date, self-care interventions such as these have yet to be trialled specifically with a population of AS patients. These may offer an appropriate, clinically effective and economically sustainable means of support for this patient cohort. Future research should aim to address this issue to ensure that psychological distress in this patient group does not go underdiagnosed and undermanaged.

\section{Author affiliations \\ ${ }^{1}$ Faculty of Medicine, Department of Primary Care and Population Sciences, University of Southampton, Southampton, UK \\ ${ }^{2}$ Division of Surgery and Interventional Science, University College London \& Department of Urology, UCLH NHS Foundation Trust, London, UK \\ ${ }^{3}$ Faculty of Health Sciences, University of Southampton \& University Hospital Southampton NHS Foundation Trust, Southampton, UK \\ ${ }^{4}$ Department of Urology, Southampton University Hospitals NHS Trust, Southampton, UK \\ ${ }^{5}$ Department of Mathematics, University of Southampton, Southampton, UK \\ ${ }^{6}$ Department of Urology, Hampshire Hospitals NHS Trust, Winchester, UK}

Contributors SW was involved in study development and management, and data analysis. GL and CE were involved in critical evaluation and academic supervision. BB was involved in critical evaluation and patient recruitment. PP was involved in statistical analysis. CMM was involved in patient recruitment and critical evaluation. AR was involved in study design and critical evaluation. $\mathrm{CP}$ was involved in patient recruitment, data extraction and data analysis. GL was involved in study design and academic supervision.

Funding This work was supported by the National Institute for Health Research School of Primary Care Research, grant number NSPCR 73.

\section{Competing interests None declared.}

Ethics approval Berkshire Research Ethics Committee (REC Code 11/SC/0071).

Provenance and peer review Not commissioned; externally peer reviewed.

Data sharing statement Additional data can be accessed via the Dryad data repository at http://datadryad.org/ with the doi:10.5061/dryad.8n7gf.

Open Access This is an Open Access article distributed in accordance with the Creative Commons Attribution Non Commercial (CC BY-NC 4.0) license, which permits others to distribute, remix, adapt, build upon this work non-commercially, and license their derivative works on different terms, provided the original work is properly cited and the use is non-commercial. See: http://creativecommons.org/licenses/by-nc/4.0/

\section{REFERENCES}

1. Office for National Statistics. London, UK, 2012.

2. Bray F, Lortet-Tieulent J, Ferlay J, et al. Prostate cancer incidence and mortality trends in 37 European countries: an overview. Eur $J$ Cancer 2010;46:3040-52.
3. Dall'Era MA, Albertsen PC, Bangma C, et al. Active surveillance for prostate cancer: a systematic review of the literature. Eur Urol 2012;62:976-83.

4. Wilt TJ, Brawer MK, Jones KM, et al. Radical prostatectomy versus observation for localised prostate cancer. N Engl J Med 2012;367:203-13

5. National Institute for Clinical Excellence Clinical Guideline 175 Prostate cancer: diagnosis and treatment. 2014.

6. Fawzy FL, Fawzy NW, Arndt LA, et al. Critical review of psychosocial interventions in cancer care. Arch Gen Psychiatry 1995;52:100-13.

7. Davidson JB, Degner LF. Empowerment of men newly diagnosed with prostate cancer. Cancer Nurs 1997;20:187-96.

8. Salander $\mathrm{P}$, Bergenheim T, Henriksson R. The creation and protection of hope in patients with malignant brain tumours. Soc Sci Med 1996;42:985-96.

9. Balderson $\mathrm{N}$, Towell $\mathrm{T}$. The prevalence and predictors of psychological distress in men with prostate cancer who are seeking support. Br J Health Psychol 2003;8:125-34.

10. Burnet KL, Parker C, Dearnaley D, et al. Does active surveillance for men with localized prostate cancer carry psychological morbidity? Br J Urol Int 2007;100:540-3.

11. van den Bergh RCN, Essink-Bot ML, Robol MJ, et al. Anxiety and distress during active surveillance for early prostate cancer. Cancer 2009;1:3867-78.

12. van den Bergh RCN, Essink-Bot ML, Robol MJ, et al. Do anxiety and distress increase during active surveillance for low risk prostate cancer? J Urol 2010;183:1786-91.

13. Department of Health. No health without mental health: a cross-government mental health outcomes strategy for people of all ages. London, UK, 2011.

14. Patel MI, DeConcini DT, Lopez-Corona E, et al. An analysis of men with clinically localised prostate cancer who deferred definitive therapy. J Urol 2004;171:1520-4.

15. Latini DM, Hart SL, Knight SJ, et al. The relationship between anxiety and time to treatment for patients with prostate cancer on surveillance. J Urol 2007;178:826-32.

16. Zigmond AS, Snaith RP. The hospital anxiety and depression scale. Psychiatr Scand 1983;67:361-70.

17. Maher EJ, Mackenzie C, Young T. The use of the Hospital Anxiety and Depression Scale (HADS) and the EORTC QLQ-C30 questionnaire to screen for treatable unmet needs in patients attending routinely for radiotherapy. Cancer Treat Revised 2006;22:123-32.

18. Lawrie I, Lloyd-Williams M, Taylor F. How do palliative medicine physicians assess and manage depression. Palliat Med 2004; 18:234-8.

19. Love AW, Kisssne DW, Bloch S, et al. Diagnostic efficiency of the Hospital Anxiety and Depression Scale in women with early stage breast cancer. Aust N Z J Psychiatry 2002;36:246-50.

20. Love AW, Grabsch B, Clarke DM, et al. Screening for depression in women with metastatic breast cancer: a comparison of the Beck Depression Inventory Short Form and the Hospital Anxiety and Depression Scale. Aust N Z J Psychiatry 2004;38:526-31.

21. Bellardita T, Rancati T, Alvisi MF, et al. Predictors of health related quality of life and adjustment to prostate cancer during active surveillance. Eur Urol 2013;64:30-6.

22. Lofters A, Juffs HG, Pond GR, et al. 'PSA-it is': knowledge of serum prostate specific antigen and other causes of anxiety in men with metastatic prostate cancer. $J$ Urol 2002;168:2516-20.

23. Schofield PE, Butow PN, Thompson JF, et al. Psychological responses to patients receiving a diagnosis of cancer. Ann Oncol 2003;14:48-56.

24. Watts S, Leydon G, Brich B, et al. Depression and anxiety in prostate cancer: a systematic review and meta-analysis of prevalence rates. BMJ Open 2014;4:e003901.

25. Cockle-Hearne J, Faithful S. Self-management for men surviving prostate cancer: a review of behavioural and psychosocial interventions to understand what strategies can work, for whom and in what circumstances. Psychooncology 2010;19:909-22. 